\title{
Two perceptual variables in within-event learning
}

\author{
ROBERT A. RESCORLA \\ University of Pennsylvania, Philadelphia, Pennsylvania
}

\begin{abstract}
Four autoshaping experiments employed pigeon subjects to explore within-stimulus learning. Experiments 1 and 2 investigated the formation of an association between two elements within a visual compound as a function of the similarity of the elements to each other. As in earlier experiments on the formation of associations between stimuli, similarity improved learning within a stimulus. Experiments 3 and 4 examined the formation of color-form associations when the color was a property of the form or of the ground on which the form occurred. Learning was better between the form and its color than between the form and the color of its background. These results suggest that perceptual variables contribute to within-stimulus learning.
\end{abstract}

Component stimuli in a compound conditioned stimulus (CS) form associations not only with the consequent unconditioned stimulus (US) but also with each other. Evidence for this assertion has been found in such common conditioning preparations as autoshaping in pigeons, flavor-aversion learning in rats, and conditioned suppression in rats (see Rescorla, 1981a; Rescorla \& Durlach, 1981). Moreover, such associations apparently occur in a variety of conventional Pavlovian paradigms, such as conditioned inhibition, blocking, and conditional discrimination procedures (e.g., Rescorla \& Durlach, 1981). It has been argued that these within-compound associations are important both because of their implications for interpretation of CS-US associations and because they may be prototypes of how organisms represent multifeatured events. Because of their ubiquity and potential importance, it is of interest to determine the rules by which such associations are formed.

Previously we have reported some evidence on variables affecting the formation of within-compound associations. For instance, they are formed rapidly, but show an orderly growth as a function of the number of exposures (e.g., Rescorla \& Durlach, 1981). More interestingly, they apparently are especially strong when the components of the compound occur simultaneously, rather than sequentially. This observation has led us to suggest a kind of perceptual notion of such learning, according to which a unitary representation of the compound event is formed (e.g., Rescorla, 1981a). This view, in turn, suggests that certain perceptual variables not commonly studied in Pavlovian experiments might affect the formation of within-compound associations.

The present report describes investigations of two variables emphasized by the Gestalt psychologists as having an important impact on our perception of objects: stimu-

This research was supported by NSF Grants BNS-78-02752 and BNS83-08176. The author's address is: Department of Psychology, University of Pennsylvania, 3815 Walnut Street, Philadelphia, PA 19104. lus similarity and figure-ground relations. The question of interest is whether the circumstances known to affect perceptual organization and segregation of events likewise affect the formation of within-event associations. Experiments 1 and 2 explored the importance of similarity among components of a compound. Experiments 3 and 4 examined the role of figure-ground relations. All experiments employed sensory-preconditioning-like procedures embedded in an autoshaping paradigm with pigeon subjects. In such procedures, the birds are first exposed to a compound stimulus (either reinforced or not), then given Pavlovian conditioning or extinction with one component, and finally tested with the other component. Withincompound associations are assessed in terms of the degree to which a pecking response to the second component depends on the treatment of the first.

\section{EXPERIMENT 1}

Rescorla and Furrow (1977) reported that associations were formed especially rapidly between two stimuli in a second-order autoshaping design if those stimuli were similar to each other. In that experiment, the birds first received discriminative first-order conditioning in which two stimuli (one color and one line orientation) were paired with food, whereas two others (another color and line orientation) were not. Then the latter stimuli were paired with the former in a second-order paradigm in such a way that for one group similars were paired, whereas for the other group dissimilars were paired. The pairing of similars produced more rapid learning.

Rescorla and Furrow's (1977) experiment used sequential presentation of stimuli with which the animal had previously received extensive separate experience. For this reason it is most naturally thought of as demonstrating that similarity promotes the formation of associations between perceptually distinct events. But it is of interest to ask whether similarity would also promote learning when the component stimuli lack independent histories 
and are presented simultaneously. Is within-event learning likewise facilitated by this perceptual relation?

Experiment 1 investigated this question by using a sensory preconditioning procedure in which the birds were first exposed to two stimulus compounds, each consisting of the simultaneous spatially adjacent presentation of two components. The component stimuli were colors and line orientations arranged so that for one group color-color and orientation-orientation compounds were given, whereas for the other group each color was paired with a line orientation. So that the association could be assessed, each bird received discriminative autoshaping in which a component of one compound was paired with food, whereas a component of the other was nonreinforced. Finally, performance to the other components was tested.

\section{Method}

Subjects and Apparatus. The subjects were 16 naive female Carneaux pigeons about 1 year old, maintained at $75 \%$ of their freefeeding weights.

The apparatus included four identical operant chambers, each measuring $27 \times 27 \times 35 \mathrm{~cm}$. The metal front panel of each chamber had a $5 \times 5 \mathrm{~cm}$ food magazine in its center, located $5 \mathrm{~cm}$ above the wire mesh floor. Three response keys, $2.5 \mathrm{~cm}$ in diameter, were located one directly above the hopper and one on either side of the center of the front wall, $20 \mathrm{~cm}$ above the floor. Located behind the right-hand key was an IEE in-line projector that permitted the transillumination of the key with four elemental stimuli. Two of these were colors (blue [B] and yellow [Y]) and two were orientations of black grids on white backgrounds (either $45^{\circ}$ or $135^{\circ}$ from the vertical). The $B$ and $45^{\circ}$ stimuli could be projected on the top half of the key; $\mathrm{Y}$ and the $135^{\circ}$ orientation could be projected on the bottom half. When any component was presented separately, the remainder of the key was black; when both portions of the key were illuminated, a $1-\mathrm{mm}$ black line separated the components. In addition, the entire key could be illuminated by a uniform white stimulus (W). These stimuli were generated by Ektachrome slides of drawings composed of Color-aid artist's paper. The remaining walls and the ceilings of the chambers were composed of clear Plexiglas.

These chambers were placed in sound- and light-attenuating shells with ventilation fans providing background noise of $62 \mathrm{~dB}$ re $20 \mu \mathrm{N} / \mathrm{m}$. On the rear wall of these shells was mounted a 6-W bulb that was continuously illuminated during the session, except during the operation of the food hopper. The hopper contained Purina Pigeon grain.

Procedure. The birds were initially magazine trained by being placed in the chambers with the hoppers activated. After the birds began eating, the hoppers were activated for progressively shorter times at longer intervals until the birds ate regularly during a 5-sec presentation. On the next 2 days all birds received 445 -sec hopper presentations, spaced a mean of 1 min apart.

On each of the next 7 days, all birds received simple autoshaping. Each session contained 245 -sec presentations of W, each terminating in $5 \mathrm{sec}$ of grain. The mean intertrial interval was $2 \mathrm{~min}$.

On each of the next 3 days, the birds were exposed to 12 nonreinforced presentations of each of two compound stimuli. For the 8 birds in Group Similar, the components of each compound were similar to each other. One compound consisted of $\mathrm{B}$ and $\mathrm{Y}$ and the other of the two grids. The 8 birds in Group Dissimilar received the same components re-paired so as to generate dissimilar compounds. In order to maintain the birds' orientation toward the key, 6 reinforced presentations of $W$ were intermixed with these 24 non- reinforced compound trials. Trials in this and all subsequent phases of the experiment occurred at a mean rate of one per minute.

On each of the next 3 days, the birds received a treatment intended to produce autoshaping of one component of one compound. Half of the birds in each group received 12 reinforced presentations of $\mathrm{Y}$ and 12 nonreinforced presentations of the $135^{\circ}$ grid. Half received the same keylights with the reinforcement contingencies reversed.

The next session assessed the consequence of these treatments for responding to $\mathrm{B}$ and the $45^{\circ}$ grid. The session began with six appropriately reinforced trials on each of the stimuli differentially treated in the previous phase. It continued with six nonreinforced presentations each of $\mathrm{B}$ and the $45^{\circ}$ grid. The question of interest was whether responding to the latter stimuli would be governed by the treatment of their associates and whether that effect would be stronger in the animals receiving similar compounds.

\section{Results and Discussion}

Autoshaping of $\mathrm{W}$ resulted in a moderate level of responding. The mean response rate on the day prior to the introduction of the compounds was 51 responses per minute. Little responding occurred to the compound stimuli when they were introduced. The mean response rates for the compounds and $\mathrm{W}$ on the final day of compound exposure were 3 and 48 responses per minute, respectively. Differential responding rapidly developed to $Y$ and the $135^{\circ}$ grid during the next phase. On the third day of discrimination training the mean response rates were 44 and 1 for the reinforced and nonreinforced component, respectively.

The data of most interest, from the test session with $B$ and the $45^{\circ}$ grid, are displayed in Figure 1. For each group, the data are displayed separately for the component with the reinforced associate $(+)$ and the component with the nonreinforced associate ( - ). Overall, there was greater responding to the associate of a reinforced stimulus [Wilcoxon $T(16)=1, p<.01$ ]. The sensitivity of a component to the treatment of the stimulus with which it had previously been compounded provides evidence for the presence of within-compound associations. This result agrees with previous results on within-compound learning in autoshaping (e.g, Rescorla, 1981b). Of more interest, the difference in responding was greater in Group Similar. This was evident both in the greater level of responding to the associate of the reinforced element [Mann-Whitney $U(8,8)=4, p<.05$ ] and in a superior discrimination ratio in Group Similar $[U(8,8)=2$, $p<.02]$. This outcome suggests that a more substantial within-compound association was formed when the components were similar to each other.

Although the overall evidence for within-compound associations seems secure, it is possible that the apparently greater responding in Group Similar is at least partially attributable to stimulus generalization. For instance, the animals that received $\mathrm{B} / \mathrm{Y}$ and $45^{\circ} / 135^{\circ}$ followed by $\mathrm{Y}+$ and $135^{\circ}-$ may have responded to $B$ both because of the $B-Y$ association and because of the generalization from $Y$. Comparable animals in Group Dissimilar received $45^{\circ} / \mathrm{Y}$ and $\mathrm{B} / 135^{\circ}$ followed by $\mathrm{Y}+$ and $135^{\circ}-$. Their association between the $45^{\circ}$ grid and $\mathrm{Y}$ may also have been 


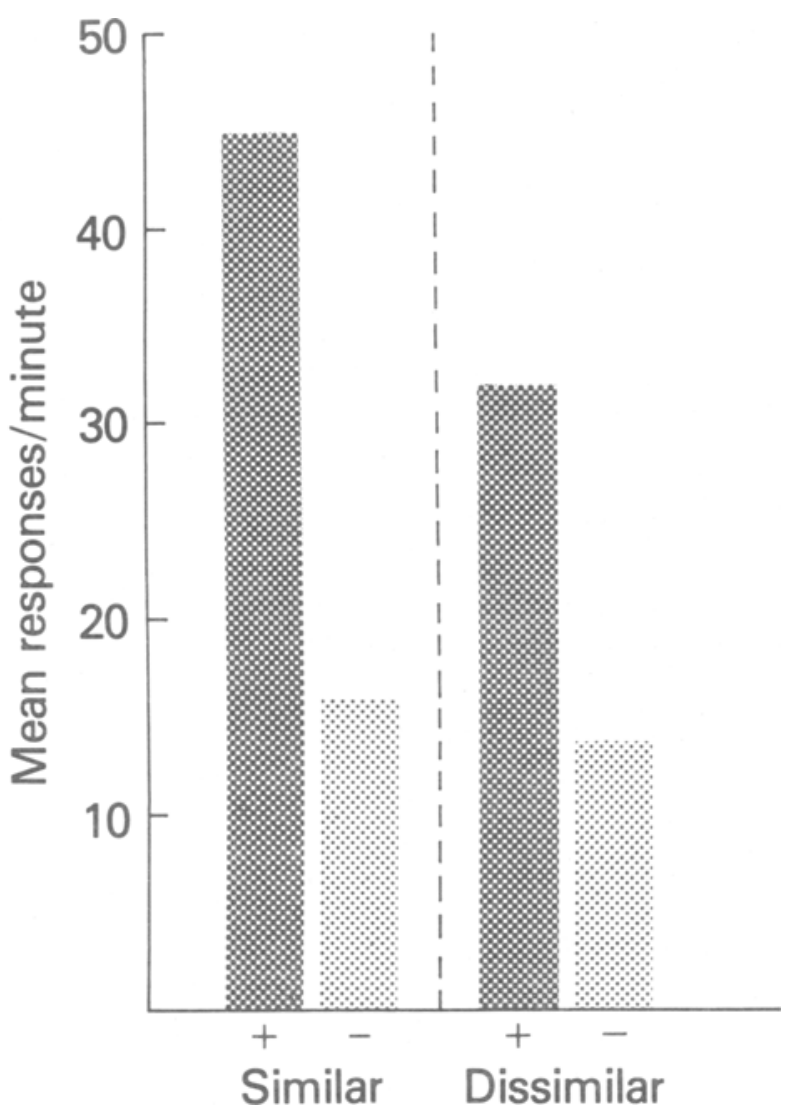

Figure 1. Mean responses per minute during the test session of Experiment 1. Responding is shown separately for the associate of the reinforced $(+)$ and nonreinforced $(-)$ stimulus for groups receiving the pairing of similars and dissimilars.

strong, but $Y$ may have generalized less to the grid than to $B$. This account correctly anticipates greater responding to the positive stimulus in Group Similar but it also predicts greater responding to the negative stimulus in Group Dissimilar. For instance, the above-described Group Dissimilar animals had B as the associate of the negative stimulus, whereas for the Group Similar animals, $45^{\circ}$ played that role. When $Y$ was then reinforced, stimulus generalization should have been greater to the associate of the negative stimulus in Group Dissimilar (B) than to that in Group Similar $\left(45^{\circ}\right)$. That difference is not present in the data. Nevertheless, it would be useful to have an assessment of the impact of similarity on withincompound associations that did not admit of this alternative. Experiment 2 provided such a demonstration.

\section{EXPERIMENT 2}

The intention of this experiment was to compare the magnitude of within-compound associations in Groups Similar and Dissimilar under conditions of comparable opportunities for stimulus generalization. To accomplish this, the procedure of Experiment 1 was modified so that both $\mathrm{Y}$ and the $45^{\circ}$ stimulus were paired with food after subjects' exposure to the compounds. The animals first were exposed to $\mathrm{B} / \mathrm{Y}$ and $45^{\circ} / 135^{\circ}$ (Group Similar) or to $\mathrm{B} / 135^{\circ}$ and $45^{\circ} / \mathrm{Y}$ (Group Dissimilar) and then received $\mathrm{Y}+$ and $135^{\circ}+$. With this procedure, $\mathrm{B}$ and $45^{\circ}$ should receive comparable stimulus generalization from $\mathrm{Y}$ and $135^{\circ}$ in the two groups. Differential responding could then be attributed to the different strengths of their within-compound associations. This reasoning is identical to that used by Rescorla and Furrow (1977) except that in the present case compound presentation preceded, rather than followed, first-order conditioning of one element.

\section{Method}

Subjects and Apparatus. The subjects were 16 female Carneaux pigeons that had previously participated in other autoshaping experiments using different stimuli and a different response key. Their assignment to groups in the present experiment was arranged to be orthogonal to their past treatment. The apparatus was that used in Experiment 1.

Procedure. Because these birds had previous autoshaping experience, no magazine training was necessary. Otherwise they were treated as were the birds in Experiment 1, except that both $Y$ and $135^{\circ}$ were reinforced after compound training. Initially, all animals received 7 days of autoshaping with $W$. During each day the 5 -sec exposure to $\mathrm{W}$ terminated in $5 \mathrm{sec}$ of grain; trials were given at a mean rate of one per $2 \mathrm{~min}$.

On each of the next 3 days, the animals received 12 nonreinforced presentations of two compounds, intermixed with 6 reinforced presentations of $W$. For the 8 animals of Group Similar the compounds were $\mathrm{B} / \mathrm{Y}$ and $45^{\circ} / 135^{\circ}$; for those in Group Dissimilar they were $\mathrm{B} / 135^{\circ}$ and $45^{\circ} / \mathrm{Y}$. In these sessions, and throughout the remainder of the experiment, the mean intertrial interval was $1 \mathrm{~min}$.

On each of the next 3 days, all birds received 12 reinforced presentations each of $\mathrm{Y}$ and the $135^{\circ}$ grid. Finally, all animals received a single test session. That session began with 6 reinforced presentations each of $\mathrm{Y}$ and $135^{\circ}$ and continued with 6 nonreinforced presentations each of $\mathrm{B}$ and $45^{\circ}$. The question of interest was whether responding to $B$ and $45^{\circ}$ would be greater in Group Similar.

\section{Results and Discussion}

Initial exposure to $\mathrm{W}$ and the compounds produced results similar to those of Experiment 1. By the final day of compound exposure, the mean rates of responding were 51 and .5 responses per minute for $\mathrm{W}$ and the compounds, respectively. Responding rapidly developed to $\mathrm{Y}$ and $135^{\circ}$ when they were reinforced. By the final day of that training, the mean rate of responding to those stimuli was 71 per minute.

The data of most interest are from the test session with $B$ and the $45^{\circ}$ stimulus. During that session the mean response rate for Group Similar was 52 responses per minute, whereas that for Group Dissimilar was 24 responses per minute. The superiority of responding in Group Dissimilar was statistically reliable [Mann-Whitney $U(8,8)$ $=12, p<.05]$.

The results of this experiment thus confirm those of Experiment 1 , that responding to a component of a compound is greater when it is similar to its associate. Experiment 2 had the advantage of exhibiting this effect under conditions that match stimulus generalization. Con- 
sequently, Experiment 2 identified an effect of similarity upon within-compound associations that occurs in addition to whatever contribution is made by stimulus generalization. However, because this was accomplished by reinforcing an element of both compounds, the opportunity to measure within-compound associations against a control treatment was necessarily sacrificed. This comparison was provided by the design of Experiment 1 .

\section{EXPERIMENT 3}

This experiment explored another perceptual variable, figure-ground relations. Perceptual evidence suggests that when a figure appears on a background, properties of the figure seem more related to each other than to properties of the ground. Moreover, Asch (1969) presented evidence that this perception markedly affects memory. Using human subjects, he found that a superior association was formed between a shape and color when the color was perceived as a property of the shape rather than of the ground on which the shape appeared. This experiment asked whether similar considerations affect the formation of within-compound associations in autoshaping.

Birds were presented with color-form compounds composed in two ways: an outline form was presented on a response key with a color confined to the form or on a key on which the color extended over the whole key, both inside and outside of the form. To the human observer, the former display gave the impression of a colored form (e.g., a red triangle) on a white background, whereas the latter appeared as an outline form superimposed on a colored field (e.g., an outline triangle on a red key). The question of interest was whether the color-form association would be superior in the former case.

In this experiment, each animal was presented with two colors (red and green) and two outline forms (square and triangle) in such a way that each form was paired with each color. However, one form appeared as an outline with one color as the background and as a form with the other color confined to its interior; the other form appeared with the colors reversed. Thus some animals had a triangle presented sometimes with a green interior and other times on a red field; those same animals had a square presented sometimes with a red interior and other times on a green field. As a result, each form was presented with each color, but under differing perceptual conditions. This design ensures comparable treatment of the individual colors and forms and provides a sensitive within-subjects comparison for the strength of the various associations. Following this exposure, half the animals received reinforced presentations of red and nonreinforced presentations of green; half received the reverse. Finally, all animals were tested for their responses to the forms. To the degree that a stronger association is formed when the color is confined to the form, the above-described animals should show more responding to the triangle when green was reinforced and more responding to the square when red was reinforced. For those animals green had been the interior color for the triangle but the background color for the square, whereas red had had the opposite roles.

\section{Method}

Subjects and Apparatus. The subjects were 16 female Carneaux pigeons with histories like those of the birds in Experiment 2. The apparatus was that of Experiment 2 modified so that the right-hand key permitted the presentation of color and form stimuli. The forms were a triangle and a square measuring $15 \mathrm{~mm}$ on a side, constructed in outline form by a 1-mm black line. These forms could appear superimposed on a red or green illumination of the full response key, or on a white key with the color confined to the interior of the form.

Procedure. Because the subjects had previously participated in other experiments, no magazine training was necessary. On each of the first four sessions, all birds were given six 5-sec exposures to each of the four color-form compounds, all nonreinforced. For half the birds, those compounds were a red triangle, a green square, an outline triangle on a green background, and an outline square on a red background. For the other half of the birds, the roles of the colors were reversed. Throughout the experiment, the mean intertrial interval was $1 \mathrm{~min}$.

On each of the next 5 days the birds were given discrimination training with the two colors. Half of the birds in each of the abovedescribed groups received 125 -sec presentations of red, each terminating in $5 \mathrm{sec}$ of grain, and 125 -sec nonreinforced presentations of green. The other half received the reverse reinforcement contingencies. The colors consisted of illumination of the full response key.

On the next day the animals were tested for their responses to the forms. That session began with 12 additional discrimination trials with the appropriate reinforcement contingencies and continued with 6 nonreinforced presentations of each outline form superimposed on a white background. The question of interest was whether responding would be greater to the form whose interior color had been reinforced.

\section{Results and Discussion}

During preexposure there was little responding to the compounds. Discrimination between the colors developed rapidly when they were differentially reinforced. By the final day of this phase, the mean response rates were 41 and 6 responses per minute for the reinforced and nonreinforced color, respectively. The data of most interest are from the testing of the forms. During that test the mean response rate for the form whose interior color had been reinforced was 24 per minute; that for the form whose background had been reinforced was 8 per minute (Wilcoxon $T=1, p<.05)$. Since the form whose interior color had been reinforced was also paired with a different background color that had been extinguished, this result suggests that the interior color exercised more control over responding to the color than did the background color. That is, a stronger association appears to have formed between a form and its interior color.

It is worth noting that the manner in which the color was displayed during its pairing with the US was most similar to the manner in which the color was displayed as a background. In both cases, coloring of the full key was involved. Inasmuch as such similarity aids the organism in ascertaining that the color actually paired with a form has been paired with the US, this should favor 
changes in responding to the form that had been presented on the colored background. For this reason, it is possible that the present results underestimate the superiority of the association formed when the color is restricted to the interior of the form.

\section{EXPERIMENT 4}

This experiment attempted to replicate the results of Experiment 3 , using a different assessment technique. For this purpose, a somewhat less frequently used procedure was adopted for detecting the color-form associations. In this procedure (see Rescorla \& Cunningham, 1978, Experiment 2) the compounds are initially reinforced and then one element is separately extinguished. Associations between elements are identified by the loss in responding that such extinction produces in the other element. For this experiment, the compounds used in Experiment 3 were followed by reinforcement and then one color was extinguished. To the degree that the association of the extinguished color is greater with one form than with the other, we anticipate greater loss in responding to that form.

\begin{abstract}
Method
Subjects and Apparatus. The subjects were 16 female Carneaux pigeons that had previously participated in another autoshaping experiment employing a different response key and different stimul. The treatments of the present experiment were arranged to be orthogonal to those of the animals' past. The apparatus was that used in Experiment 3.

Procedure. On each of the first 4 days, the birds were given six 5-sec presentations of each of four color-form compounds, each terminating in $5 \mathrm{sec}$ of grain. The compounds were the same as those used in Experiment 3, balanced across animals in the same manner. On each of the next 3 days the animals received 245 -sec nonreinforced presentations of the key illuminated fully by one of the colors. For half of the animals this was green; for the other half it was red.

On the next day, responding to the forms was tested. This session began with 12 additional nonreinforced presentations of the appropriate color, followed by 6 nonreinforced presentations of each form on a white background. The question of interest was whether responding would be less to the form whose interior color had been extinguished.
\end{abstract}

\section{Results and Discussion}

Responding developed to a moderate level for all of the compounds. By the final day of training the mean response rate was $\mathbf{5 7}$ per minute. Responding rapidly extinguished to a level of 2 responses per minute by the last day of nonreinforcement of the colors. The data of most interest are from the test of the forms. The mean response rate was 7 per minute to the form whose interior color had been extinguished and 22 per minute to that whose background color had been extinguished $[T(12)=8$, $p<.05]$. This difference suggests a greater decremental effect on responding to a form when its interior, rather than its background color, was nonreinforced. This is consistent with the proposition that a stronger association was formed with the interior color.

\section{GENERAL DISCUSSION}

The results of the present experiments demonstrate that two perceptual variables are contributors to the strength of within-compound associations. Memory for the internal organization of a stimulus is apparently promoted by variables that encourage perceptual organization. This finding accords with the general theoretical position of Gestalt psychology and the particular empirical findings of Asch (1969). It is consonant with a recent description of the between-stimuli learning that occurs in Pavlovian conditioning as heavily influenced by perceptual variables (Rescorla, 1985).

The findings with regard to similarity of component stimuli are directly analogous to results that indicate that between-events learning is promoted by similarity (e.g., Rescorla \& Furrow, 1977). They join other results that indicate that intrinsic stimulus relations between stimulus events assist the formation of associations. Not all stimuli appear to be equally associable with each other; rather, there are inherent relations that impact the formation of associations. The best-known results of this sort are the so-called cue-to-consequence findings from the flavoraversion literature. However, in the present instance, the kind of informative relation between events that would lead one to identify one stimulus as a "cue" and one as a "consequence" is lacking. Such intrinsic relations apparently matter within as well as between events.

The figure-ground results also have an analogy in between-event learning. The present method of arranging for a color to be property of the background can be described as adding to a colored figure further coloring outside of the figure. This technique was used deliberately so that the extent to which the color was spatially contiguous with the form would not differ between the two treatments. But the technique of presenting additional color outside of the form is a kind of spatial analogue to a procedure that is important to current theories of the temporal relation between stimuli in Pavlovian conditioning. Several authors (e.g, Durlach, 1983; Gamzu \& Williams, 1973; Jenkins, Barnes, \& Barrera, 1981; Rescorla, 1968) have reported that a CS-US contiguity that is otherwise capable of producing conditioning can be made ineffective if USs are added at times other than during presentation of the CS. This result is important because it suggests that it is CS-US contingency, rather than simple contiguity, that promotes conditioning. The treatments of Experiments 3 and 4 can be viewed as making a similar suggestion for spatial relations within an event. Apparently, a form-color spatial contiguity can be made less effective if the spatial contingency is reduced by presenting the color in places outside of the form. In both cases the manipulation can be viewed as reducing the perceptual relation between the components without changing the degree to which they are contiguous. It remains to be determined whether the sort of formal theory that has been proposed to account for the case of a degraded temporal relation between events (e.g., Rescorla \& Wagner, 
1972) can be extended to deal with the degraded spatial relation within an event.

The present data can also be accommodated within an interpretation of within-compound learning that is nonassociative. Rescorla and Durlach (1981) suggested that some instances of such learning can be viewed as the organism's forming a unitary representation of the compound. When individually presented, the components can then activate that representation because of their similarity to it. For instance, in a sensory preconditioning procedure, presentation of an $\mathrm{AB}$ compound may leave a representation that both $A$ and $B$ could activate. When $B$ is then reinforced, it reactivates that representation so that it is paired with the reinforcer. Subsequent test presentations of $A$ similarly activate the $A B$ representation and produce a response because of the association between $\mathrm{AB}$ and the US. In this case, conditions that lead to perceptual unity might facilitate formation of a unitary representation. Such a view receives some encouragement from Lamb and Riley's (1981) interpretation of perceptual effects on another measure of memory, matching-tosample tasks.

It is also possible that the particular mode of stimulus presentation affects the relative salience of the elements of a compound. For instance, a shape placed on a uniform ground may be less salient than one whose internal color differs from the ground. The resulting difference in salience could affect a shape's ability to enter into an association with a contiguous color, or its relative importance in a unitary representation.

Whether one adopts an associative or nonassociative view of within-compound learning, it is important to realize that the operation of perceptual variables must receive more molecular explanation. Molar perceptual concepts may provide useful analogies for understanding learning about individual events and associations among those events; but these analogies are only a first step in identifying the action of variables, not an explanation of that action.

\section{REFERENCES}

Asch, S. E. (1969). Reformulation of the problem of association. American Psychologist, 24, 92-102.

DURLACH, P. J. (1983). Effect of signaling intertrial unconditioned stimuli in autoshaping. Journal of Experimental Psychology: Animal Behavior Processes, 9, 374-389.

Gamzu, E. R., \& Williams, D. R. (1973). Associative factors underlying the pigeon's key pecking in autoshaping procedures. Journal of the Experimental Analysis of Behavior, 19, 225-232.

Jenkins, H. M. Barnes, R. A., \& Barrera, F. J. (1981). Why autoshaping depends on trial spacing. In C. M. Locurto, H. S. Terrace, \& J. Gibbon (Eds.), Autoshaping and conditioning theory. New York: Academic Press.

LAMB, M. R., \&ILEY, D. A. (1981). Effects of element arrangement on the processing of compound stimuli in pigeons (Columba livia) Journal of Experimental Psychology: Animal Behavior Processes, 7, 45-58.

Rescorla, R. A. (1968). Probability of shock in the presence and absence of CS in fear conditioning. Journal of Comparative \& Physiological Psychology, 66, 1-5.

Rescorla, R. A. (1981a). Simultaneous associations. In P. Harzem \& M. Zeiler (Eds.), Advances in analysis of behavior (Vol. 2). New York: Wiley.

RescorLa, R. A. (1981b). Within-signal learning in autoshaping. Animal Learning \& Behavior, 9, 245-252.

Rescorla, R. A. (1985). Pavlovian conditioning analogues to Gestalt perceptual principles. In F. R. Brush \& J. B. Overmier (Eds.), Affect, conditioning, and cognition: Essays on the determinants of behavior. Hillsdale, NJ: Erlbaum.

Rescorla, R. A., \& Cunningham, C. L. (1978). Within-flavor associations. Journal of Experimental Psychology: Animal Behavior Processes, 4, 267-275.

Rescorla, R. A., \& Durlach, P. J. (1981). Within-event learning in Pavlovian conditioning. In R. R. Miller \& N. E. Spear (Eds.), Information processing in animals: Memory mechanisms. Hillsdale, $\mathrm{NJ}$ Erlbaum.

ResCorla, R. A., \& FURROw, D. R. (1977). Stimulus similarity as a determinant of Pavlovian conditioning. Journal of Experimental Psychology: Animal Behavior Processes, 3, 203-215.

Rescorla, R. A., \& Wagner, A. R. (1972). A theory of Pavlovian conditioning: Variations in the effectiveness of reinforcement and nonreinforcement. In A. Black \& W. F. Prokasy (Eds.), Classical conditioning II. New York: Appleton-Century-Crofts.

(Manuscript received March 24, 1986; revision accepted for publication August 29, 1986.) 